\title{
UNA RELECTURA DE LOS NOVÍSIMOS DESDE LA POESÍA DE LOS ‘80. EL CASO DE VÍCTOR BOTAS
}

\author{
Marta Beatriz Ferrari \\ Universidad Nacional de Mar del Plata
}

En los treinta y seis años que han pasado desde la aparición de la Antología de Castellet Nueve Novísimos poetas españoles, hemos podido asistir a una intensa discusión crítica que ha venido comprometiendo no sólo a muchos de los poetas en ella incluidos, sino también a un sinnúmero de críticos y de poetas no antologados que no quisieron quedar al margen de una polémica que se suscitó al menos en dos frentes: uno de naturaleza cuasi historiográfica y otro de ribetes estético/ideológicos. Por una parte, desde la efectiva aparición de Nueve Novísimos, la reflexión crítica se ha ido decantando a favor o en contra del concepto de "novedad" y de "ruptura" que la antología implicaba. Muchas fueron las voces que sostuvieron la existencia de un corte epistemológico en el paradigma poético español hacia mediados de la década del ' 60 ; muchas otras, en cambio, se erigieron en contradogma limitando la impronta rupturista que, desde sus comienzos, y, refrendada por la crítica y el mundo universitario, pretendió definir a esta estética. En este sentido, Victor García de la Concha, subrayaba muy tempranamente que muchas de las características aglutinantes del grupo «ya se venían anotando en promociones anteriores» (1990: 112), y resultaba asimismo sintomático el planteo con que abría el encuentro de poetas y críticos celebrado en Oviedo, en 1985, en torno a si existía o no una verdadera renovación en el caso de los "Novísimos" y "si éstos inducían un tiempo dialéctico nuevo en la poesía española tal como Castellet pretendía» (1986: 55). En esta misma línea, se puede incluir la tesis de Manuel Rico quien hablará de una "ruptura escalonada" (57-64) preparada, en rigor, por algunos de los mismos poetas sociales del '40 y del '50 (José Hierro con su Libro de las Alucinaciones sería un claro cjemplo), una escritura en la que ya alienta la renovación luego atribuida en exclusividad a los novísimos. De similares opiniones serán José Luis Falcó y Fanny Rubio, Enrique Martín Pardo y Juan José Lanz, quien en su minucioso estudio sobre la "generación del '68" plantea el tema en términos de «cambio evolutivo" o "conciencia y voluntad de ruptura» (22.3), abonando de este modo la tesis de la evolución natural y la continuidad con los modos poéticos del '50 y el ' 60 . Por su parte, Ángel Prieto de Paula llamará la atención sobre la interesada parcialidad que suele 


\section{UNA RELECTURA DE LOS NOVÍSIMOS DESDE LA POESÍA DE LOS ‘ 80}

revelar la construcción que de la historia literaria han hecho algunos protagonistas del episodio novísimo que pretendieron cxhibir como novedad lo que sólo era «naturalización de actitudes y procedimicntos anteriores» (373-390).

Hoy ya no resulta tan difícil hallar el suficiente sustento conceptual para cuestionar el supuesto carácter rupturista con que irrumpe la escritura "novísima" en el panorama poético de los " 70 . Por una parte, si se atiende al enlace del grupo con los poetas del ' 50 o ' 60 , se podrá notar que la inclusión de miembros de uno y otro grupo en las tempranas antologías de Martín Pardo (1967) y de Batlló (1968), auténticos anticipos de Nueve novísimos, habla a las claras de la pacífica convivencia de las voces de Pedro Gimferrer y Manuel Vázquez Montalbán con la mayoritaria presencia de los poetas del medio siglo. Del mismo modo, el año de publicación de Arde el mar de Gimferrer (1966) coincide, por ejemplo, con la aparición de Moralidades de Gil de Biedma, mientras que Dibujo de la muerte de Guillermo Carnero (1967), lo hará con Tratado de urbanismo de Ángel González, datos que impiden sostener que el asentamiento de la nueva estética tuvo lugar sobre las ruinas de la estética precedente. Por otra parte, la conceptualización misma de la ruptura (esa nueva voluntad de entroncar la poesía española con una tradición que ahora venía de Europa y también de EE. UU.) estaba hallando su cauce de expresión en los diversos ensayos teóricos que ya a comienzos del ' 60 hablaban, como lo haría José Ángel Valente, de la distancia que va de la "tendencia" al "estilo" en poesía (26-29) o, como lo haría también Gil de Biedma en torno a la relación entre poesía y comunicación (17-31). Polémica a la que se sumaron las voces de Carlos Bousoño seguida por la lúcida réplica de Carlos Barral en su artículo "Poesía no es comunicación", de $1953^{1}$.

El segundo frente de discusión se articula casi exclusivamente en torno a la conflictiva interpretación del giro autorreferencial que exhibe gran parte de la escritura del '70. Al llegar a este punto, la crítica discurrió una vez más por vías antagónicas. Así, nos encontramos con posturas tan radicales como la de Carlos Bousoño, por ejemplo, quien considera que «detrás de todo texto metapoético existe la voluntad de desmitificar el lenguaje restrictivo del poder» (176), las declaraciones de Andrew Debicki, quien con algunas reservas adhiere a la tesis de Bousoño (43-44), o la voz más reciente de Juan José Lanz quien se refiere a "la estética comprometida" de los poetas novísimos; protesta que, según su análisis, «se hace sutil, esquiva, resbaladiza» para mejor sortear los mecanismos represores de la censura, «modos de compromiso indirecto» que afectaban fundamentalmente al lenguaje y se cifraban en «la problematización de las relaciones entre palabra y realidad práctica» (8-13).

En el extremo opuesto, sin embargo, Joan Oleza y Jenaro Talens, defendieron la tesis de que el discurso metapoético venía a reforzar el carácter autónomo del texto. Oleza y José Luis Angeles, por un lado, señalaron que el Formalismo y el Estructuralismo crearon "el sueño de la autosuficiencia del lenguaje" y asignaron a esta tendencia ese volverse de la poesía sobre sí misma: «En una ceremonia propia de la ameba - dicen- la poesía se secuestraba a sí misma y el poeta excavaba gozosas trincheras tras las que parapetarse de la vida. Bousoño dijo que ese volverse de espaldas del lenguaje a la vida era un gesto de rebeldía, pero esto, a estas alturas del pensamiento teórico, resulta una broma, o lo que es peor, una coartada» $(248-9)^{2}$. Fue precisamente el término "coartada" el que empleó Jenaro Talens para referirse a la práctica metapoética de los "Novísimos"; y afirmó que la ambigüedad con que se abordó la cuestión de la función social de la poesía de los setenta partió de la utilización que se ha hecho del ambiguo concepto de

1.- A la definición de Bousoño «poesía es la transmisión puramente verbal de una compleja realidad anímica previamente conocida por el espíritu, como formando un todo, una síntesis» (Teoría de la expresión poética), responde Barral: «ello supone la preexistencia al poema de un contenido psíquico que pudiera ser explicado idiomáticamente, y que es transmitido al lector [...], a la manera romántica sería ese contenido preexistente al poema el clemento sustancial de la emoción poética». ("Poesía no es comunicación”, Revista Laye, 23, 1953).

2.- Cabe señalar que esta expresión procede del ensayo de Fernando Savater Panfleto contra el todo. 


\section{MARTA BEATRIZ FERRARI}

"metapoesía" propuesto por Bousoño. Talens señala que la función operativa del término no fue definir una forma de escritura sino más bien «dar por sentada la existencia de una poesía que no fuese al mismo tiempo metapoesía, esto es, aceptar la posibilidad de existencia de lo que Derrida llamaría una metafísica de la presencia, es decir, de algo que está ahí y de lo que nosotros nos limitamos a hablar» (55); concepción de lo poético que emergería como deudataria del programa romántico-becqueriano según el cual el poeta sería, una vez más, el depositario de ese "algo" intangible e inexpresable.

Estamos frente a una tendencia poética que despertó polémicos cuestionamientos de todo signo y a diferentes niveles. El carecer de programas o manifiestos de agresión y defensa, el haber constituido un grupo de efímera cohesión lleva a Guillermo Carnero a afirmar que pertenece a «una promoción de poetas entre los que no hay afinidad ni intercambio de ninguna clase» (89). De hecho, muchos fueron los que abandonaron rápidamente la causa original y muchos también los que cambiaron de género para intentar la narrativa, el guión cinematográfico o la crítica literaria. En este sentido, el abandono del castellano como lengua poética por parte de Pedro Gimferrer precisamente en 1970, puede ser interpretado como un modo de deserción efectiva de la estética novísima, contribuyendo así a la tesis de Ignacio Prat para quien «el año fundacional de la antología es también su año funeral» (76). Problemática que se extendió a quiénes eran, estrictamente, los poetas comprendidos bajo esta etiqueta generacional, quedando, en principio, planteado así el problema de la segmentación dentro de la serie literaria. Visto con suficiente perspectiva histórica, hoy podemos advertir, por ejemplo, que junto con el episodio novísimo existió un grupo de poetas bifrontes que revelaron un doble entronque, tanto con las estéticas precedentes como con las que acabarían imponiéndose a comienzos de los '80. En su ensayo Literatura y generaciones (1975), Julián Marías proponía el concepto de "constelación" para definir precisamente a una agrupación de autores que aparecen como una unidad generacional pero que, de hecho, pertenecen a dos, y ejemplificaba: "Cuando alguien es muy precoz, aparece incorporado a la generación anterior y su "edad social" parece mayor que la real; por el contrario, cuando alguien es tardío -al menos en su manifestación pública-resulta socialmente más joven y se incorpora a la generación siguiente, formando "constclación" con ella, aunque en el fondo pertenezca radicalmente a la que le corresponde».

Ya en 1980, José Luis García Martín reunía en Las voces y los ecos a una serie de poetas nacidos después del '39 y que hubiesen publicado su primer libro en la década del '70, poetas excluídos de la Antología de Castellet, pero que, sin embargo, formaban "constelación" con la generación precedente; un segundo momento dentro de la promoción del '68, una transición que cristalizaría hacia 1977 su programa de renovación estética y que estaría representada por los llamados "poctas ocultos" en palabras de Luis Antonio de Villena o los "románticos solitarios" en la expresión de Luna Borge, grupo al que pertenecerían, entre otros, Abelardo Linares, Fernando Ortíz, Javier Salvago, Francisco Bejarano y Víctor Botas. Unos poetas que, en principio, no adhiricron a la faceta más conocida de la estética dominante, sino que anticiparon algunas de las líneas más reveladoras de lo que sería la poesía española de las últimas décadas.

Lo que resulta evidente a más de 30 años de aquel fenómeno, en gran medida, publicitario y surgido con una clara pretensión canónica es que los Novísimos establecieron una ruptura predominantemente formal, y lo hicieron con fuertes apoyos críticos y editoriales desde Cataluña. Antología netamente metropolitana que, sin embargo, se pretendió imponer como representativa de todo el panorama poético español de la década del '70, selección que no en todos los casos operó sobre un material previo como lo demuestran, por ejemplo, los pocmas compuestos ad hoc por Manuel Vázquez Montalbán ${ }^{3}$, Nueve Novísimos aparece hoy como un eslabón más dentro de

3.- Nos referimos al poema "Ivonne de Carlo", escrito especialmente para la Antología de Castellet y luego incluido en el pocmario Liquidación de restos de serie. 


\section{UNA RELECTURA DE LOS NOVÍSIMOS DESDE LA POESÍA DE LOS ‘ 80}

aquel movimiento cultural que tuvo lugar en la Barcelona de los años ' 60 , y que parecía llamado a renovar las concepciones estéticas y sociológicas de la cultura dentro de un proyecto general de modernización política, que acabaría rompiendo con la inercia impuesta por el franquismo ${ }^{4}$.

¿Es ésta la generación de Víctor Botas? Por simple cronología biográfica -nace el mismo año que Pedro Gimferrer-, Botas pertenecería no problemáticamente a la promoción de los '70. Sin embargo, su primer libro de poemas, Las cosas que me acechan', se publica nueve años después de la aparición de la Antología de Castellet, cuando la etapa más decididamente "novísima" de muchos "novísimos" ya había sido superada. Nos encontraríamos, entonces, más próximos a libros como Scholia de Luis Alberto de Cuenca (de 1978) o Huir del invierno de Luis Antonio de Villena (escrito entre el 77 y el 81), poetas estos últimos incorporados por Antonio Prieto a su Antología Espejo del Amor y de la Muerte, en 1971. Pero este primer poemario de Botas se aleja de los cánones estéticos dominantes anunciando en más de un sentido algunas de las direcciones más representativas de los ' 80 . Se trata aquí de breves composiciones medidas (con una decidida preferencia por el endecasílabo), cuyo abanico temático se despliega insistentemente sobre el amor, el tiempo y la muerte, entrecruzando la problemática existencial de acentos quevedianos con la metapoética; declaración de una fe vacilante en la poesía, más en la línea de José Hierro que en la de Guillermo Carnero. Una escritura en la que la huella borgiana resulta más que evidente (como en muchos poetas del '80, pienso en Felipe Benítez Reyes), evidente no sólo en lo que comporta a selección léxica y modos de construcción del poema (la acumulación enumerativa, por ejemplo), sino especialmente en los motivos asociados al tema de la identidad, como el del laberinto, los espejos, el doble, el otro que es el mismo. Pocmas enunciados (como en los sociales del "40) desde un "yo" todavía confesional, con un correlato biográfico identificable, un sujeto a la deriva, definido por la perplejidad, la incertidumbre y el miedo, un hombre que yerra en la literatura y en la vida y que se emparenta directamente con el sujeto menor de Angel González, el de Áspero mundo, por ejemplo, aquel que se definía como el excedente, fragmento y sustancia desechable, nunca el centro sino los márgenes, resto, escombro, poeta mendicante que clama por la limosna de una palabra. Poemas dirigidos siempre a un "tú" ausente, que es sólo posibilidad, que es sólo deseo, en un mundo desprovisto de certezas y gobernado por el caos y el azar. Pero justo es decir que en este primer libro están preanunciados ya el correlato objetivo, la máscara cultural de Prosopon, su poemario siguiente, e incluso, cierto aire oriental, de budismo zen, de sus composiciones posteriores.

Ahora bien, si hubo algo que más o menos aglutinó a las variadas manifestaciones escriturales de los '70, eso fue la omnipresencia de discursos culturales de diverso signo en el corpus poemático, hasta el punto de quedar cristalizados en cl calificativo de "culturalistas"; una escritura que apelaba a todo el bagaje cultural posible en respuesta al empobrecimiento del lenguaje propiciado por

4.- Cfr. Amparo Amorós, "Los Novísimos y cierra España! Reflexión crítica sobre algunos fenómenos estéticos que configuran la poesía de los años ochenta", Insula, 512-513, agosto-septiembre 1989, p. 63. La llamada escuela de cinematografía de Barcelona estaba formada por gente muy influenciada por la Nouvelle Vague francesa: Pere Portabella, Carlos Duran, Jacinto Estevan, Ricardo Bofill, Joaquim Jordà, Gonzalo Suárez, Jaime Camino, Jorge Grau, José M. Nunes, Teresa Gimpera y Serena Vergano, entre otros. Entre los poetas forman parte de este grupo Jaime Gil de Biedma, José Agustín Goytisolo, Carlos Barral, Alfonso Costafreda, Jaime Ferrán, L.orenzo Gomis y Enrique Badosa. Bajo la advocación de Machado, la Escuela de Barcelona va a hacer su presentación en sociedad con la publicación en 1960 de la antología de Josep M. Castellet, Veinte años de poesía española. Nacidos entre 1925 y 1929, son autores que se vinculan a una doble tradición lírica: autores catalanes como Riba o Carner y en castellano. Son hombres de cultura cosmopolita que leyeron y tradujeron a Auden, Eliot, Yeats, Rilke, Pasolini, Pavese, Baudelaire. Procedían de familias bien acomodadas y tenían formación universitaria; unidos por profundos lazos de amistad fueron los iniciadores de lo que sería la ruptura con la inercia cultural impuesta por el franquismo. 5.- La numeración de las páginas corresponde en todos los casos a la siguiente edición: Botas, Víctor, Poesía Completa. Gijón: Llibros del Pexe, 1999. 


\section{MARTA BEATRIZ FERRARI}

las estéticas de mensaje exclusivamente social. El propio Gimferrer explicaba por esos mismos años: «escribimos poesía por mimetismo», pero aclaraba: «mímesis no de la realidad, sino de la cultura» ${ }^{6}$. La tantas veces repetida formación masmediática de los miembros de esta generación no soslaya, sin embargo, el deliberado entronque que los mismos establecieron con una tradición que bien puede arrancar del simbolismo, prolongarse en el modernismo y alcanzar la vanguardia surrealista en algunos nombres del '27, especialmente Aleixandre, Lorca, Cernuda y a través de este último, la poesía romántica alemana e inglesa. Consciente de la importancia de evitar el impudor romántico, lo que él mismo denominó "el vicio de la falacia patética" o el engaño sentimental, Cernuda será un pionero en el camino de objetivar la experiencia vivida de un modo similar a como lo harían años más tarde, Jaime Gil de Biedma, Luis Antonio de Villena o Luis García Montero.

Pero, quizá, dentro del mismo grupo novísimo original ya estuvieran perfiladas las dos subtendencias que hoy, cuando leemos a estos poctas del ' 70 desde los ' 80 , reconocemos con tanta facilidad. Mientras en Gimferrer (por tomar el caso más paradigmático del grupo) el empleo del collage sigue la línea sígnica y visual de Pound o Eliot, es decir, que su función sería iconográfica y aséptica, en Vázquez. Montalbán, en cambio, el mismo recurso responde a un afán democratizador de la cultura y se carga de connotaciones ideológicas, acompañando este proceso con la introducción del lenguaje coloquial y del registro irónico. En él la descontextualización de los materiales permite una lectura metonímica de esos textos que, aunque disímiles, pasan a formar parte de una misma serie y como consecuencia, posibilita una formulación sintética de los opuestos.

Paralelamente a las apropiaciones literarias, muchos textos de esta primera línea señalada incorporan y tematizan motivos pictóricos, escultóricos, históricos o musicales. Los poemas surgen como un segundo lenguaje redundante, como una reduplicación de una experiencia estética ya plasmada en una obra artística previa. El referente del poema vuclve a ser un producto cultural; se crea así un circuito cerrado, un círculo de referencias indefinidas que no logra trascender los límites de un universo definido estéticamente. La vinculación con "lo real" parece si no imposible, al menos no declaradamente necesaria. Esta elección, que supone un cierre de puertas a la realidad extratextual, subraya la voluntad de autonomía estética de estas escrituras al hacer del poema un universo autosuficiente. La estetización que propugnan estos discursos implica un acto sustitutorio de la vida por el arte (vienen a la memoria los emblemáticos versos de Carnero: «raso amarillo a cambio de mi vida», o de Gimferrer: "os doy mi vida a cambio de un pendiente de plata»). Las declaraciones de Gimferrer resultan, una vez más, incontestables:

Nunca he hecho poesía comprometida porque a mí me interesa escribir para los que Stendhal llamó the happy few'. Lo escribí al final de Fortuny y la gente se molestó pero creo que son el auténtico público de la literatura. A la larga es el núcleo reducido el que decide. La expansión de un autor siempre se da a partir de las minorías, de un grupo formado por snobs y expertos. (13)

Estos poctas que se reconocen como sabios artífices del verso, oficio reducido al de unos pocos hombres ilustrados; son -como ellos mismos se han designado-poetas del "Mester de Clerecía". Textos sólo para iniciados y cuya exitosa decodificación supone, en muchos casos, la proyección de una continuidad de sentido sobre la discontinuidad de la representación, la poesía novísima produjo, así, un discurso que desestimó el realismo, se rebeló contra la expresión directa del "yo" y contra el lenguaje referencial, y quebró deliberadamente el pacto de lectura.

La relectura de la tradición que hace Botas difiere radicalmente de la que en su momento hicieron los Novísimos; en ocasiones se trata de los mismos objetos pero de diversos modos de

6.- Citado por Fanny Rubio en "Hacia una constitución de una poesía española en castellano. Un lustro desasosegado (Propuesta ficción)". El Estado de las Poesías. Los Cuadernos del Norte. Monografía Nro. 3, Caja de Ahorros de Asturias, 1986. 


\section{UNA RELECTURA DE LOS NOVÍSIMOS DESDE LA POESÍA DE LOS ‘ 80}

leer. Muy alejada del empleo frío y despolitizado del collage que hicera Gimferrer, por cjemplo, la escritura intertextual del poeta asturiano (cl acento puesto siempre en la emoción y la inteligibilidad del texto) se aproxima más a la intencionalidad que orienta al recurso en Vázquez. Montalbán (pienso en Las coplas a la muerte de mi tía Daniela, por ejemplo), en quien la reescritura paródica de los clásicos se lee dentro del amplio espectro que cubre la operación desmitificadora de la "alta literatura canonizada". No se trata necesariamente de recuperar moldes vacíos de significación, también en los clásicos se busca la emoción, que posibilita la proximidad y la complicidad con el lector,

Ahora bien, en los ' 80 (década a la que pertenece la mayor parte de la obra poética de Botas) ya no hay lagunas culturales que llenar porque la cultura es algo a lo que se puede acceder muy fácilmente y la poesía española está ya plenamente integrada al concicrto de las literaturas del resto de Europa y América. La cultura y sus objetos dejan de ser el referente obligado del poema; se rompe así el círculo tautológico y el gesto narcisista que redireccionaba la lectura hacia la materialidad misma de la escritura. La de los " 80 es una poesía que abre sus puertas a la "realidad", si bien como señalará Jon Juaristi, se trata de «una convención intersubjetiva que consiste en un acuerdo sobre los efectos de realidad del poema, no sobre los recursos para producirlo ${ }^{7}$, y en la histórica dicotomía entre arte y vida, esta última obtiene una clara ventaja; Miguel García Posada titulará precisamente "Del culturalismo a la vida" a su estudio preliminar a los llamados poetas de la experiencia (17-29). En cuanto a los procedimientos escriturales se produce un regreso a los esquemas métricos y estróficos, al "yo" omnipresente, al lenguaje referencial y a la expresión transitiva y no problemática de los contenidos de conciencia, estrategias todas que apuntan a renovar el perdido pacto de lectura.

Hace poco más de diez años, Darío Villanueva realizaba estas declaraciones refiriéndose al contexto de la literatura española de las últimas décadas:

Si siempre escribir significó imitar los modelos precedentes, en el fecundo equilibrio entre tradición y originalidad, ello ha cobrado nueva vigencia en la cscritura "palimpsestuosa" -por remedar el conocido libro de Genette sobre 'la literatura en segundo grado'- característica de esta época cenital en la que, como Umberto Eco ha reconocido, la vanguardia se ha convertido en tradición y ya no cabe ser escritor (ni lector) adánico, ya no se puede defender ni restaurar la ingenuidad (27). ${ }^{8}$

La cita de Villanueva insiste en el tópico posmoderno de que todo está ya dicho y/o escrito y que a diferencia del momento de las vanguardias, cuando existía la opción radical de destruir para inventar-romper con la historia, el pasado, la tradición-, al lector y al escritor de nuestra época ya no le quedan más opciones que re-visitar ese pasado pero sin la ingenuidad del primer hombre y asumiendo conscientemente el peso de esa historia". Borges en su cuento "Pierre Menard, autor del Quijote", del año 1939, ya se servía de la imagen del palimpsesto, cuando afirmaba respecto de

7.- Juaristi, Jon, "El pacto realista", Insula, 565 (enero 1994), p. 26.

8.- Conviene aclarar que el adjetivo "palimpsestuosa" procede, tal como lo señala Genette, de Philippe Lejeune.

9.- El pasaje de Umberto Eco al que se refiere Darío Villanueva es el siguiente: «la respuesta posmoderna a lo moderno consiste en reconocer que, puesto que el pasado no puede destruirse -su destrucción conduce al silencio-, lo que hay que hacer es volver a visitarlo con ironía, sin ingenuidad. Pienso que la actitud posmoderna es como la del que ama a una mujer muy culta y sabe que no puede decirle "Te amo desesperadamente", porque sabe que ella sabe (y que ella sabe que él sabe) que esas frases ya las ha escrito Liala. Podrá decir: "Como diría Liala, te amo desesperadamente”. En ese momento, habiendo evitado la falsa inocencia, habiendo dicho claramente que ya no se puede hablar de manera inocente, habrá logrado sin embargo decirle a la mujer lo que quería decirle: que la ama, pero que la ama en una época en que la inocencia se ha perdido. Si la mujer entra en el juego, habrá recibido de todos modos, una declaración de amor. Ninguno de los interlocutores se sentirá inocente; ambos habrán aceptado el desafío del pasado, de lo ya dicho que es imposible eliminar; ambos jugarán a conciencia y con placer el juego de la ironía... Pero ambos habrán logrado una vez más hablar de amor». Eco, Umberto, Apostillas a El nombre de la Rosa. Barcelona: Lumen, 1986. p. 74-75. 


\section{MARTA BEATRIZ FERRARI}

la empresa llevada a cabo por Menard, reconstruir literal $\mathrm{e}$ imposiblemente, la obra de Cervantes: «He reflexionado que es lícito ver en el Quijote "final" una especie de palimpsesto, en el que deben traslucirse los rastros -tenues pero no indescifrables- de la "previa" escritura de nuestro amigo» (450).

A mediados de la década del '70, Harold Bloom publicaba un ensayo central para examinar la historia de la lectura y escritura poética a partir de lo que él denominó una nueva "inquietud" o "desasosiego" literario (términos con los que se interpretó su concepto de "anxiety") producido por la constatación de que se ha llegado tarde al mundo, y que ya todo ha sido dicho por nuestros padres poéticos, nuestros antepasados o precursores. En este libro, La angustia de las influencias, el ensayista se preguntaba, entre otras cosas, «¿Qué son las influencias poéticas después de todo? ¿Podría ser su cstudio realmente algo más que la tediosa labor de búsqueda de fuentes, del recuento de alusiones, una labor que muy pronto ha de llegar al apocalipsis de todos modos cuando pase de las manos de los eruditos a las máquinas calculadoras?» $(41-42)^{10}$. Y se empeñaba en subrayar: «Es necesario que dejemos de pensar en cualquier poeta como si fuera un cgo autónomo. Todo poeta es un ser atrapado en una relación dialéctica $[\ldots]$ con otro u otros poetas».

En la escritura de Víctor Botas la superficie textual revela un minucioso tratamiento reconstructivo del pasado; una operatoria intencionada y selectiva que nos confirma, una vez más, que cuando leemos, siempre leemos, como mínimo, dos textos. Después de Prosopon, libro que tematiza el mundo familiar con un estilo algo más coloquial y en el que todavía, en la dialéctica arte/vida, hay un claro saldo a favor de esta última, su siguiente pocmario, Segunda Mano, nos enfrenta a una concepción de la escritura como reescritura. El libro es un viaje por la historia de la literatura, un recorrido que avanza desde la escritura más antigua de la humanidad (el Poema de Gilgamesh) hasta mediado el siglo XX y cuyo hilo conductor vuelve a ser una insistente meditación sobre la muerte, sobre la impermanencia del tiempo, del amor, de la poesía. Es, junto con Historia Antigua, el libro de las variaciones: el lenguaje poético concebido como interrupción y reinicio de un diálogo que se ha roto por siglos o sólo por un instante. No se trata de repetición, ni de reproducción de un acontecimiento pasado, se trata, más bien, de una mímesis paradójica por la que no se obtiene una copia sino una variación, en el sentido musical del término, en que la misma melodía es planteada bajo un número muy amplio de formas. La repetición (pensemos en las traducciones casi literales de "Los dos poemas ingleses" de Borges) no es la puesta en presente de lo pasado, sino la transformación de las categorías del tiempo cronológico repitiendo, en todo caso, el gesto deseperado de Menard, el fracaso o la imposibilidad misma de la repeticion.

Por su parte, el registro irónico de Aguas mayores y menores, que desacraliza escatológicamente la tradición clásica -los epigramas cróticos de la Antología palatina pero también el utillaje retórico modernista, el mito romántico de la inspiración y la pedantería culturalista-, emparenta a esta escritura con la de los '80. Del mismo modo, en Historia Antigua (1987), uno de los mejores libros de poesía de la década, en opinión de José Luna Borge (106), las marcas de oralidad, el humor y la narratividad de los poemas que, a modo de contrapunto, van alternando escenas de la Historia (con mayúsculas) con escenas de la vida cotidiana, anticipan en más de diez años muchos de los poemas de Luis Alberto de Cuenca, sobre todo, los de Por fuertes y fronteras.

10.- En este libro, cuyo título nos remite al poemario de W. H. Auden The Age of Anxiety (1947), Bloom aclara que no le interesa la acepción de "influencias poéticas" reducida a «transmisión de ideas e imágenes de los poetas anteriores a los posteriores» (85), puesto que unas y otras pertenecen, como él señala, a la discursividad y a la historia. De ahí que se dedique a indagar en las muchas modalidades de "influencia": desvío, completación, ruptura, demonización, privación ascética, celebración del retorno. En la escritura poética de Botas, sin embargo, el entusiasmo por las influencias, tal como iremos viendo a lo largo de este trabajo, parece excluir todo sentimiento de angustia; podríamos, incluso, calificar a su poesía como exhibicionista, en el sentido de que el padre poético nunca se oculta sino que se muestra orgullosamente. 
El concepto de tradición y el de diálogo intertextual con la tradición resulta, muchas veces, más productivo que el de generación, puesto que, en la práctica, cada generación literaria realiza su propia lectura de la tradición; rescatando y postergando nombres según afinidades y gustos, más o menos azarosos, más o menos condicionados, van conformando un nuevo canon estético. El mismo Víctor Botas declaraba algo semejante: «No creo en las rupturas de tipo cultural; en todo caso lo que hay son variaciones, pequeños pasos, a partir siempre de una tradición previa que, eso sí, puede estar, en ocasiones, muy lejana en el tiempo. En tal sentido, considero más adecuado hablar de gustos dominantes en un momento dado, modas, o cualquier otra expresión; pero no de ruptura» (280).

En su escritura, Botas hace suyo aquel aforismo de La Bruyére sobre el que Guillermo Carnero compusiera sus propias variaciones: "Todo está ya dicho y hemos llegado demasiado tarde»; dice Botas: "y tú,/ tú seguirás aquí,/ consumiendo ese tiempo que a ti mismo,/ a su vez, te consume;/ colocando/ palabras que no van/ a ser leídas nunca/ nunca,/ porque no dicen nada/ que no hayan repetido muchas voces/ muertas/ que los demás se saben de memoria» (242). Hablar por boca de otros, escribir entre comillas, citar, aún no sabiendo que citamos, y sobreescribir un poema infinito que nadie alcanzará a leer sino a pedazos.

\section{Referencias bibliográficas}

BLOOM, Harold (1991): La angustia de las influencias. Caracas: Monte Ávila Latinoamericana. BORGES, Jorge Luis (1978): Obras Completas. Buenos Aires: Emecé.

BOUSOÑO, Carlos (1979): "La poesía de Guillermo Carnero", prólogo a Ensayo de una Teoría de la Visión. Madrid: Hiperión.

CARNERO, Guillermo (1986): "Poética”, en El Estado de las Poesías. Los Cuadernos del Norte. Monografía n. ${ }^{\circ}$ 3. Caja de Ahorros de Asturias.

DEBICKI, Andrew (1989): "Una poesía de la postmodernidad: los Novísimos", Anales de la Literatura Española Contemporánea, 14.

GARCÍA DE LA CONCHA, Víctor (1986): "La renovación estética en los años sesenta", en El Estado de las Poesías. Los Cuadernos del Norte. Monografía n. ${ }^{\circ}$ 3. Caja de Ahorros de Asturias.

— _ y A. Sánchez Zamarreño (1990): Letras Españolas 1976-1988. Madrid: Castalia.

GARCÍA MARTíN, José Luis (1980): Las Voces y los Ecos. Gijón: Júcar.

GARCÍA POSADA, Miguel (1994). "Del culturalismo a la vida”, en El lugar de la poesía. Luis Muñoz (ed.). Diputación Provincial de Granada.

GIL DE BIEDMA, Jaime (1980): "Función de la poesía y función de la crítica, por T. S. Eliot". El pie de la letra. Ensayos 1955-1979. Barcelona: Crítica.

GIMFERRER, Pedro (1995): "Sólo me interesa escribir para una minoría de esnobs y expertos". $A B C, 24$ de marzo.

JUARISTI, Jon. (1994): "El pacto realista”. Insula, 565 (enero).

LANZ, Juan José (2002): "Himnos del tiempo de las barricadas: sobre el compromiso en los poetas novísimos". Insula 671-672. Noviembre-diciembre.

— (s. f.): Introducción al estudio de la Generación Poética Española de 1968. Elementos para la elaboración de un marco histórico-crítico en el período 1962-1977. Servicio Editorial de la Universidad del País Vasco.

LUNA BORGE, José (1991): La generación poética del '70. Cuestión de perspectiva. Sevilla: Qüasyeditorial. 


\section{MARTA BEATRIZ FERRARI}

OLEZA, Joan y Angeles, José Luis (1992): “La recepción de Miguel Hernández en la poesía española de los años 70 y 80 ", en Miguel Hernández, cincuenta años después. Alicante, Elche, Orihuela.

PRAT, Ignacio (1982): "Contra ti (notas de un contemporáneo de los novísimos)". Granada: Don Quijote.

PRIETO DE PAULA, Ángel (2002): "Sobre la poesía y el estatuto de la poesía en el año 2000". Diablotexto, 6.

RICO, Manuel (1992): "El acceso a la contemporancidad de la pocsía española. Las claves de una ruptura escalonada". Cuadernos Hispanoamericanos, 508, octubre .

TALENS, Jenaro (1989): "La coartada metapoética". Insula, 512-513, agosto-septiembre.

VALENTE, José Ángel (1994): Las palabras de la tribu. Barcelona: Tusquets.

VILLANUEVA, Darío (1992): "Los marcos de la literatura española (1975-1990): esbozos de un sistema" en F. Rico, Historia y Crítica de la Literatura Española. Vol. IX. Barcelona: Crítica. 